\title{
Growth of a self-assembled monolayer decoupled from the substrate: nucleation on-command using buffer layers
}

\author{
Robby Reynaerts, Kunal S. Mali and Steven De Feyter
}

\author{
Full Research Paper \\ Address: \\ Department of Chemistry, Division of Molecular Imaging and \\ Photonics, KU Leuven, Celestijnenlaan 200F, B-3001 Leuven, \\ Belgium \\ Email: \\ Kunal S. Mali* - kunal.mali@kuleuven.be; Steven De Feyter ${ }^{*}$ - \\ steven.defeyter@kuleuven.be \\ * Corresponding author \\ Keywords: \\ benzoic acid; nucleation; self-assembly; solution-solid interface; \\ substrate effect
}

Beilstein J. Nanotechnol. 2020, 11, 1291-1302.
https://doi.org/10.3762/bjnano.11.113

Received: 13 May 2020

Accepted: 12 August 2020

Published: 01 September 2020

This article is part of the thematic issue "Molecular assemblies on surfaces - towards physical and electronic decoupling of organic molecules".

Guest Editor: M. Stöhr

(C) 2020 Reynaerts et al.; licensee Beilstein-Institut. License and terms: see end of document.

\begin{abstract}
Structural polymorphism is ubiquitous in physisorbed self-assembled monolayers formed at the solution-solid interface. One of the ways to influence network formation at this interface is to physically decouple the self-assembled monolayer from the underlying substrate thereby removing the influence of the substrate lattice, if any. Here we show a systematic exploration of self-assembly of a typical building block, namely 4-tetradecyloxybenzoic acid at the 1-phenyloctane-graphite interface in the presence and in the absence of a buffer layer formed by a long chain alkane, namely $n$-pentacontane. Using scanning tunneling microscopy (STM), three different structural polymorphs were identified for 4-tetradecyloxybenzoic acid at the 1-phenyloctane-graphite interface. Surprisingly, the same three structures were formed on top of the buffer layer, albeit at different concentrations. Systematic variation of experimental parameters did not lead to any new network in the presence of the buffer layer. We discovered that the self-assembly on top of the buffer layer allows better control over the nanoscale manipulation of the self-assembled networks. Using the influence of the STM tip, we could initiate the nucleation of small isolated domains of the benzoic acid on-command in a reproducible fashion. Such controlled nucleation experiments hold promise for studying fundamental processes inherent to the assembly process on surfaces.
\end{abstract}

\section{Introduction}

The ability of some molecules to crystalize in more than one type of packing - a property widely known as crystal polymorphism - is not limited to the realm of bulk (3D) crystals but also extends to the 2D world of physisorbed self-assembled monolayers [1-4]. In fact, observation of multiple polymorphic networks, especially for organic monolayers formed at the solu- 
tion-solid interface, is a more of routine occurrence than an exception. Such structurally diverse monolayers are typically formed on solid substrates such as highly oriented pyrolytic graphite (HOPG), graphene, and metals such as $\mathrm{Cu}, \mathrm{Ag}$ and $\mathrm{Au}$ and have been characterized using scanning probe methods, especially scanning tunneling microscopy (STM) [2]. While the formation of structurally diverse crystalline monolayers provides exciting opportunities for surface modification and also for investigating crystal engineering in 2D [5], predicting $2 \mathrm{D}$ polymorphism is often nontrivial. The understanding of this already enigmatic process is further impaired by the nature of the solution-solid interface. A number of factors such as the temperature [6-9], the solvent [10-15], the substrate [16-18] and the concentration of the building block in solution [19-24] are known to influence network formation at the solution-solid interface.

One of the unconventional ways to influence the structure formation at the solution-solid interface involves the use of buffer layers. Such buffer layers typically comprise of monolayers formed by long chain alkanes [25-27] or alkane derivatives such as fatty acids [28-30] or alkylamines [31], especially when graphite is used as the substrate. The rationale here is that given the strong influence of the substrate lattice on the adsorption as well as the self-assembly of typical organic molecules, insertion of another layer in between the assembling building block and the substrate would allow the formation of an alternative polymorph that would not be obtained otherwise. In line with this strategy, self-assembled buffer layers of $n$-pentacontane $\left(\boldsymbol{n}-\mathbf{C}_{\mathbf{5 0}}\right)$ have been used to obtain a previously unknown polymorph of hexakis(n-dodecyl)-peri-hexabenzocoronene (HBC-C 12 ) which was not formed when the assembly process was carried out at the $n$-tetradecane-HOPG interface without the buffer layer [26]. Buffer layers of $\boldsymbol{n}-\mathbf{C}_{\mathbf{5 0}}$ have also been used to 'select' certain polymorphs of a Fréchet dendron based on the symmetry of the underlying alkane layer [27]. Buffer layers of tetratriacontane [25] and tridecylamine [31] were used to template the self-assembly of copper phthalocyanine. Room temperature STM measurements revealed that the adsorption as well as the diffusion of clusters of $\mathrm{CuPc}$ molecules was strongly influenced by the symmetry and the structure of the buffer layers. Notably, no self-assembly was observed when $\mathrm{CuPc}$ solutions were directly deposited on the HOPG substrate highlighting the role of buffer layers in stabilizing the self-assembled networks [25,31].

Besides their use for influencing structure formation by acting as a physical barrier between the substrate and the assembling moiety, buffer layers are also widely used to study intrinsic electronic properties of functional organic systems such as organic semiconductors $[32,33]$ and films of $1 \mathrm{D} / 2 \mathrm{D}$ polymers
[34-37] via electronic decoupling. Alkane buffer layers have been employed as efficient electronic decoupling platforms for studying the intrinsic electronic properties of graphene and fullerenes [38]. Apart from alkane derivatives, inorganic systems such as chemisorbed iodine layers [34-37], and ultrathin layers of $\mathrm{KCl}$ [39], $\mathrm{NaCl}$ [40], $\mathrm{CuN}$ [41] and oxides [32,42] have been used. Typically, the ultrathin films of these wide band gap materials act as insulating layers while still allowing electron tunneling through them. Chemisorbed iodine layers have been used as passivating layers on metals such as Au for achieving controlled electrochemical polymerization of thiophene to produce polythiophene nanowires with tunable lengths [36,37].

In the context of their use for controlling surface architectures, insertion of the buffer layers between the assembling moiety and the solid substrate affects the assembly process via the following major avenues: (1) The buffer layers, in principle, offer a new substrate with a different, often lower, symmetry. (2) The buffer layer has a different lattice constant compared to the solid substrate. (3) The adsorption enthalpy of the assembling moiety on the buffer layer is often different (lower) on the buffer layer compared to that on the solid substrate. These factors may lead to different adsorption conformation for initial single molecule adsorption coupled with higher orientational freedom and significantly different (often reduced) lateral corrugation barriers for molecular diffusion on buffer layers compared to that on pristine unmodified solid substrates. The result could be a selection of a polymorph that is otherwise not obtained under 'normal' experimental conditions. The decoupling effects could be significant especially for organic building blocks substituted with long alkyl chains because the softepitaxy of such chains with the graphite lattice is well established [43-45].

In this contribution, we present a systematic, curiosity-driven study of the self-assembly of a relatively simple building block, namely 4-tetradecyloxybenzoic acid $\left(\mathbf{B A}_{\mathbf{O}} \mathbf{O C}_{\mathbf{1 4}}\right.$, Figure 1a), with and without $\boldsymbol{n}-\mathbf{C}_{\mathbf{5 0}}$ (Figure 1b) buffer layers at the 1-phenyloctane-HOPG interface. Our previous investigation into the assembly behavior of this building block revealed that it forms structurally complex monolayers at the 1-phenyloctane-HOPG interface. Despite its simple molecular structure, the networks of $\mathbf{B A - O C} \mathbf{1 4}$ consist of a dense arrangement of hydrogen bonded dimers wherein the molecular columns show periodic kinks along the column axis after every three dimers. The origin of this relatively complex packing was thought to be the specific registry of the alkoxy chains with the substrate lattice that does not allow parallel and straight row packing of dimers due to the steric hindrance of the bulky phenyl groups. The result is a tilted arrangement of the $\mathbf{B A}-\mathbf{O} \mathbf{O C}_{\mathbf{1 4}}$ dimers that show periodic kinks that were thought to arise to maintain the 
specific registry with the substrate lattice [45]. Considering that the self-assembled network formed by $\mathbf{B A}-\mathbf{O C}_{\mathbf{1 4}}$ at the 1-phenyloctane-HOPG interface could possibly represent a substrate lattice-controlled assembly, we set out to address the following questions: (1) Will $\mathbf{B A}-\mathbf{O C}_{\mathbf{1 4}}$ form a similar network structure on top of a $\boldsymbol{n}-\mathbf{C}_{\mathbf{5 0}}$ buffer layer? (2) Would the self-assembly of BA-OC $\mathbf{1 4}$ atop the $\boldsymbol{n}-\mathbf{C}_{\mathbf{5 0}}$ buffer layer lead to the formation of another polymorph? (3) Does the assembly atop such buffer layers provide better control over our ability to monitor/manipulate dynamic assembly processes? The results and discussion provided below delve into some of these aspects.

a)

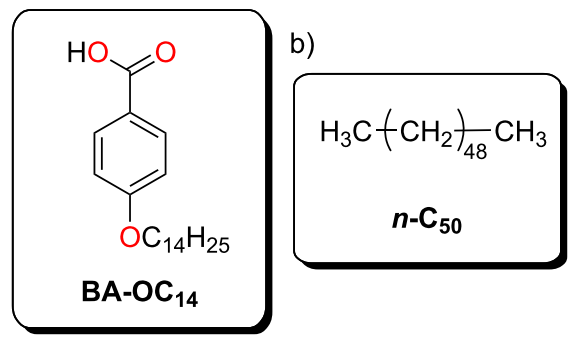

Figure 1: Molecular structures of (a) 4-tetradecyloxybenzoic acid $\left(B A-O_{14}\right)$ and $(b) n$-pentacontane $\left(n-C_{50}\right)$ used in this study. Monolayers of $\boldsymbol{n}-\mathbf{C}_{\mathbf{5 0}}$ were used as buffer layers to physically decouple the monolayers formed by $\mathbf{B A}-\mathrm{OC}_{14}$ from the underlying graphite substrate.

\section{Results and Discussion Self-assembly of BA-OC $\mathbf{C}_{\mathbf{1 4}}$ without buffer layer}

The famous and somewhat provocative quote of McCrone that, "... every compound has different polymorphic forms, and that, in general, the number of forms known for that compound is proportional to the time and money spent in research on that compound." [46] also applies to on-surface 2D assembly of building blocks. In fact, it is not unusual to observe multiple complex polymorphs for a building block with a seemingly simple molecular structure as demonstrated by the assembly of 4-octadecyloxybenzamide, which was found to form six different network structures [19]. Thus, before carrying out selfassembly experiments with $\boldsymbol{n}-\mathbf{C}_{\mathbf{5 0}}$ buffer layers, we systematically studied the concentration dependence of $\mathbf{B A}-\mathbf{O C}_{\mathbf{1 4}}$ assembly to comprehensively identify the different structures formed by $\mathbf{B A}-\mathbf{O C}_{\mathbf{1 4}}$ at the 1-phenyloctane-HOPG interface. With this exercise, we can have reasonable confidence that any 'new' polymorph observed atop buffer layers is indeed a new structure and is not formed as a consequence of slight changes in the solution concentration and thus is also observed in the absence of the buffer layer. To this effect, the concentration de- pendence of self-assembly was examined within the concentration range of $7.7 \times 10^{-4} \mathrm{M}$ to $4.0 \times 10^{-5} \mathrm{M}$. For concentrations below $4.0 \times 10^{-5} \mathrm{M}$, no self-assembly was observed.

Figure 2 shows large-scale and high-resolution STM images of the different polymorphs of $\mathbf{B A}-\mathbf{O C}_{\mathbf{1 4}}$ observed at the 1-phenyloctane-HOPG interface together with the proposed molecular models. Polymorph A (Figure 2a,d) is formed exclusively within the concentration range of $7.7 \times 10^{-4} \mathrm{M}$ to $1.9 \times 10^{-4} \mathrm{M}$. This network has been reported by us earlier [45]. The highresolution image provided in Figure 2d shows that the lamellar structure consists of bright blobs corresponding to the benzene rings flanked on either side by relatively darker regions which arise due to the tetradecyloxy chains. The benzene rings always appear in pairs indicating formation of hydrogen bonded dimers. Each lamella shows regular kinks along the lamella propagation direction. The kinks appear after every three hydrogen bonded dimers and the next three dimers are shifted with respect to the previous triplet of dimers (highlighted in red and green color, Figure $2 \mathrm{~g}$ ). The tetradecyloxy chains are oriented at $\approx 60^{\circ}$ with respect to the column axis and are always aligned along one of the main symmetry axes of the graphite lattice. The tetradecyloxy chains in the neighboring lamellae are arranged in a tail-to-tail fashion without any interdigitation. The lamellae make an angle of $\approx 8^{\circ}$ with respect to the nearest symmetry axis of graphite. The unit cell parameters of the network are provided in Table 1 and match with those reported by us earlier [45].

Lowering the concentration to $9 \times 10^{-5} \mathrm{M}$ lead to the formation of two additional networks at the 1-phenyloctane-HOPG interface. Although polymorph A remains the dominant network on the surface with the highest surface coverage $(\approx 60 \%)$, another network with a significantly different unit cell (Figure $2 b, e$ and Table 1) was observed. This network, referred hereon as polymorph B, lacks the peculiar bright-dark contrast observed in the STM images of polymorph A. Figure 2e, h shows a high-resolution STM image of polymorph B and the corresponding molecular model, respectively. It can be readily noticed that $\mathbf{B A}_{\mathbf{O}} \mathbf{O C}_{\mathbf{1 4}}$ molecules still form dimers but the molecules are stacked antiparallel to each other. The dimers show a shift in the propagation direction after every four (parallel) dimers which can be noticed from the proposed molecular model (red and green circles, Figure 2h). Due to this shift, the two (parallel) dimers with a different propagation direction are closer to each other. The molecular model also reveals that the space between four benzene rings may host a molecule of solvent adsorbed edge-on (black arrow, Figure $2 \mathrm{~h}$ ) with respect to the surface. The collection of such four benzene rings together with the co-adsorbed molecule of 1-phenyloctane is often imaged as a single bright feature in large-scale STM images (as evident 

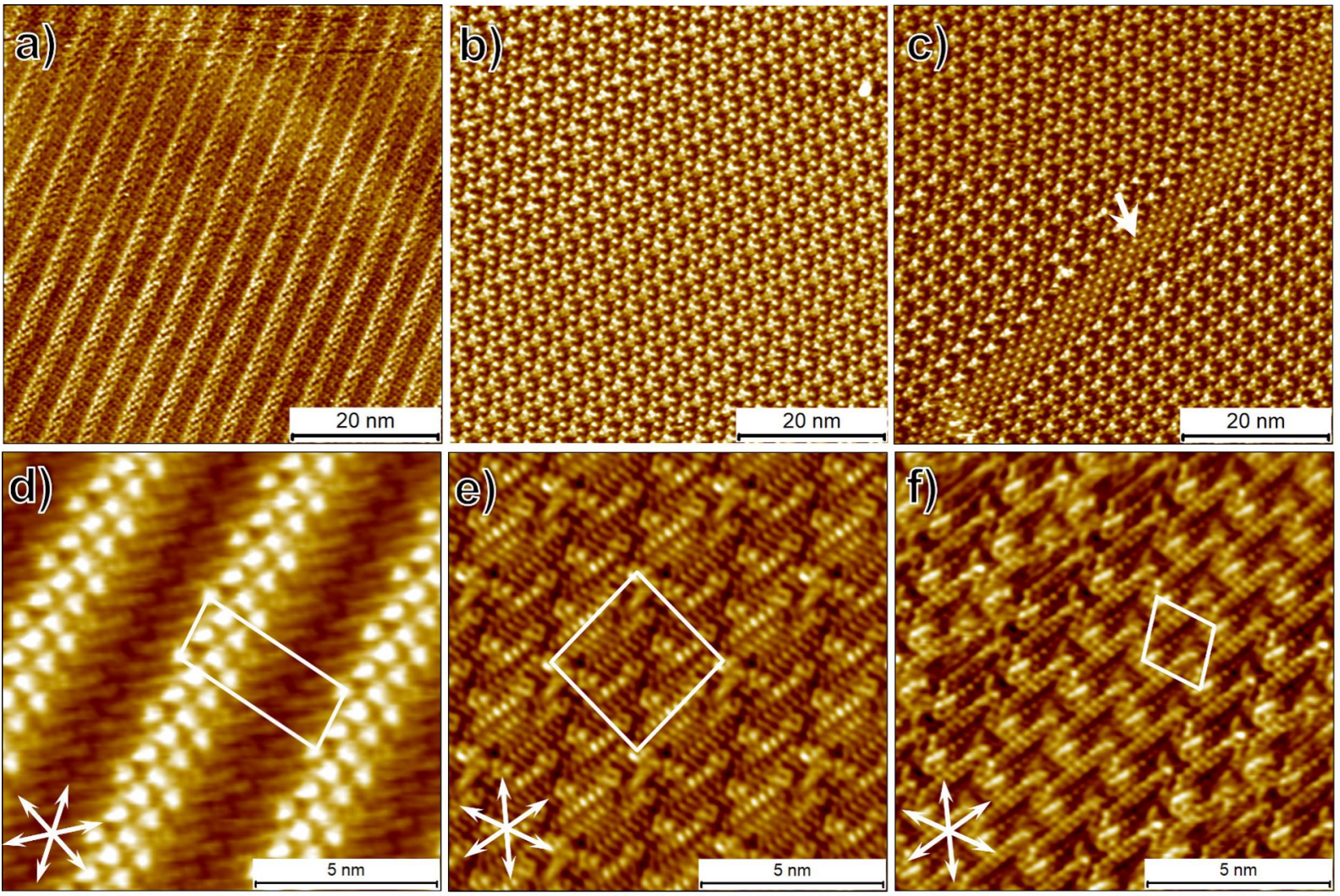

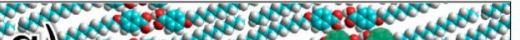
9.

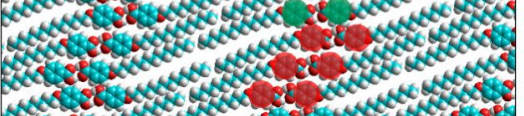

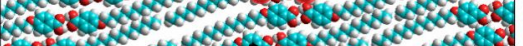
a r w w w arom 20 620

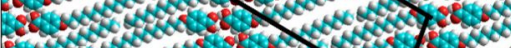
200 wat m

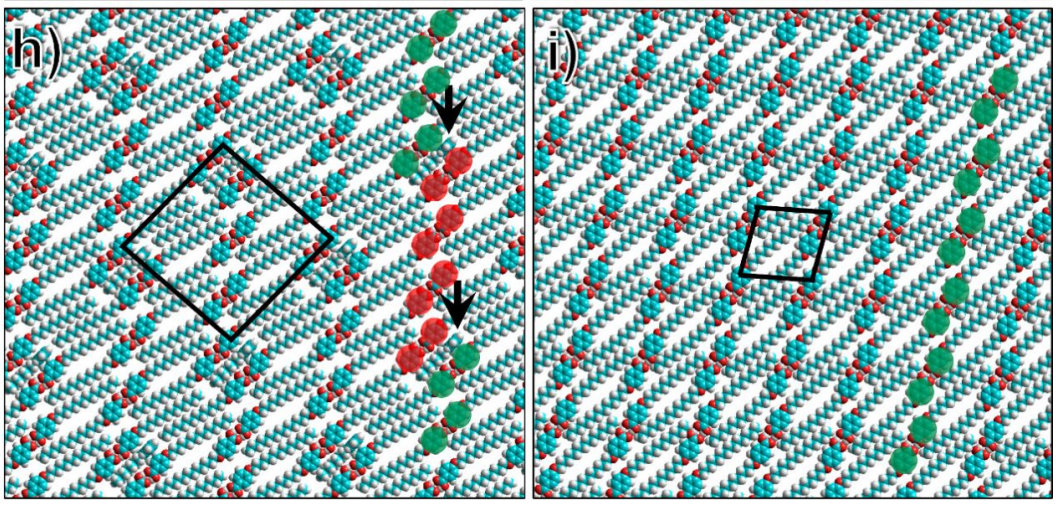

Figure 2: STM images of concentration dependent polymorphs of BA-OC $\mathbf{~}_{14}$ formed at the 1-phenyloctane-HOPG interface. (a,b,c) Large scale STM images of polymorph A, B and C, respectively. (d,e,f) High-resolution STM images of polymorph A, B and C, respectively. The white double-headed arrows at the lower left corner of the image represent the three symmetry axes of the graphite lattice. (g,h,i) Molecular models for polymorph $A$, B and $\mathrm{C}$, respectively. Imaging conditions: (a) $I_{\text {set }}=70 \mathrm{pA}, V_{\text {bias }}=-1.55 \mathrm{~V}$; (b,c) $I_{\text {set }}=140 \mathrm{pA}, V_{\text {bias }}=0.55 \mathrm{~V}$; (d) $I_{\text {set }}=50 \mathrm{pA}, V=-1.85 \mathrm{~V}$; (e,f) $I_{\text {set }}=$ $140 \mathrm{pA}, V_{\text {bias }}=0.55 \mathrm{~V}$. For additional data, see Figures $\mathrm{S} 1$ and S2 in Supporting Information File 1.

from Figure 2b). At $9 \times 10^{-5} \mathrm{M}$, the surface coverage of polymorph $\mathrm{B}$ was found to $\mathrm{be} \approx 39 \%$.

At $9 \times 10^{-5} \mathrm{M}$, another network, polymorph $\mathrm{C}$, is observed that only slightly differs from polymorph $\mathrm{B}$, and has the lowest surface coverage $(\approx 5 \%)$. Polymorph $\mathrm{C}$ (white arrow, Figure $2 \mathrm{c}$ ) tends to appear on the edges of the domains of polymorph B and can be identified by its distinct STM contrast compared to that of polymorph B. Figure 2f,i shows the high-resolution STM image and the proposed molecular model for polymorph $\mathrm{C}$, re- spectively. It is evident that the network consists of antiparallel dimers similar to those observed in the case of polymorph B, however, the dimers do not show a shift in the propagation direction (green circles, Figure 2i). Polymorph C, despite its low surface coverage was found to be stable to STM scanning and did not transform into other structures within the typical time frame of STM experiments. At concentrations lower than $9 \times 10^{-5} \mathrm{M}$ no self-assembly was observed indicating that the concentration limit for $\mathbf{B A}-\mathbf{O C} \mathbf{1 4}$ assembly under these conditions is reached. 
Table 1: Unit cell parameters for the benzoic acid polymorphs (A, B and C) observed in this study together with those of $n$ - $\mathrm{C}_{50}$ buffer layer. $D_{1}$ and $D_{2}$ are the molecular densities of the different polymorphs observed on HOPG and atop $\boldsymbol{n}-\mathbf{C}_{50}$ buffer layer, respectively. $\theta_{1}$ and $\theta_{2}$ are the percentage

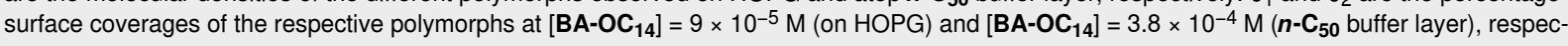
tively.

\begin{tabular}{|c|c|c|c|c|c|c|c|c|c|c|}
\hline & \multicolumn{3}{|c|}{ Unit cell (HOPG) } & \multirow{2}{*}{$\begin{array}{l}D_{1} \\
\text { (molecules/ } \\
\mathrm{nm}^{2} \text { ) }\end{array}$} & \multirow[t]{2}{*}{$\theta_{1}(\%)$} & \multicolumn{3}{|c|}{ Unit cell ( $\boldsymbol{n}-\mathbf{C}_{50}$ buffer layer) } & \multirow{2}{*}{$\begin{array}{l}D_{2} \\
\text { (molecules/ } \\
\mathrm{nm}^{2} \text { ) }\end{array}$} & \multirow[t]{2}{*}{$\theta_{2}(\%)$} \\
\hline & $\mathrm{a}(\mathrm{nm})$ & $\mathrm{b}(\mathrm{nm})$ & $\alpha\left({ }^{\circ}\right)$ & & & $a(n m)$ & $\mathrm{b}(\mathrm{nm})$ & $\alpha\left({ }^{\circ}\right)$ & & \\
\hline A & $1.8 \pm 0.1$ & $4.4 \pm 0.1$ & $81.0 \pm 2.7^{\circ}$ & 0.74 & 60 & $1.8 \pm 0.1$ & $4.6 \pm 0.1$ & $77.9 \pm 2.4^{\circ}$ & 0.71 & 50 \\
\hline B & $3.4 \pm 0.1$ & $3.4 \pm 0.1$ & $86.9 \pm 1.3^{\circ}$ & 0.68 & 39 & $3.6 \pm 0.2$ & $3.4 \pm 0.1$ & $88.2 \pm 4.9^{\circ}$ & 0.65 & 49 \\
\hline $\mathrm{C}$ & $1.7 \pm 0.1$ & $1.6 \pm 0.1$ & $79.6 \pm 1.0^{\circ}$ & 0.71 & 1 & $1.5 \pm 0.1$ & $2.0 \pm 0.2$ & $83.6 \pm 2.7^{\circ}$ & 0.68 & 1 \\
\hline$n-C_{50}$ & $6.6 \pm 0.1$ & $0.5 \pm 0.1$ & $90.0 \pm 2.0^{\circ}$ & - & - & - & - & - & - & - \\
\hline
\end{tabular}

\section{Self-assembly of BA-OC $\mathbf{C}_{\mathbf{1 4}}$ with $\boldsymbol{n}-\mathbf{C}_{\mathbf{5 0}}$ buffer layer}

Having confirmed the possibility of the formation of three different polymorphs of $\mathbf{B A}-\mathbf{O C}_{\mathbf{1 4}}$ at the 1-phenyloctane-HOPG interface, we turned our attention to the buffer layer experiments. In order to minimize the influence of the domain borders of the buffer layers on the nucleation and the growth of the BA-OC14 domains, we first optimized the deposition conditions in order to obtain large domains of $\boldsymbol{n}-\mathbf{C}_{\mathbf{5 0}}$. Specifically, we targeted domain sizes of $\geq 250 \mathrm{~nm} \times 250 \mathrm{~nm}$ by carrying out systematic concentration-dependent measurements since lower solution concentrations are known to favor large domain sizes. Annealing of the samples was also carried out. We noticed that at lower solution concentrations, the $\boldsymbol{n}-\mathbf{C}_{\mathbf{5 0}}$ monolayers exhibit a highly dynamic behavior with significant reorganization of domains upon repeated scanning with the STM tip (Figure S3 in Supporting Information File 1). Figure $3 \mathrm{a}, \mathrm{b}$ shows a typical high-resolution STM image of the $\boldsymbol{n}-\mathbf{C}_{\mathbf{5 0}}$ buffer layer together with the proposed molecular model for the assembly. $\boldsymbol{n}-\mathbf{C}_{\mathbf{5 0}}$ monolayers consist of a lamellar structure in which the alkane molecules are fully extended and are oriented at $90^{\circ}$ with respect to the lamella axis. This monolayer structure is identical to that reported earlier [26].

After thoroughly understanding and optimization of the selfassembly behavior of $\mathbf{B A}-\mathbf{O C}_{\mathbf{1 4}}$ and $\boldsymbol{n}-\mathbf{C}_{\mathbf{5 0}}$ at the 1-phenyloctane-HOPG interface, we moved on to the self-assembly experiments with the buffer layer. Although the buffer layer experiments described below were carried out using sequential deposition of solutions of $\boldsymbol{n}-\mathbf{C}_{\mathbf{5 0}}$ and $\mathbf{B A}-\mathbf{O C}_{\mathbf{1 4}}$ (in that sequence), we stress that the outcome of the experiments remained the same when carried out using premixed solutions of the two components. Typically, a drop of $1 \times 10^{-5} \mathrm{M}$ solution of $\boldsymbol{n}-\mathbf{C}_{\mathbf{5 0}}$ was first applied onto a freshly cleaved HOPG surface and the surface was imaged using STM to ensure full coverage of the $\boldsymbol{n}-\mathbf{C}_{\mathbf{5 0}}$ monolayer. After this, a drop of BA-OC $\mathbf{1 4}$ solution was applied and the imaging was resumed. Note that while choosing the concentration of $\mathbf{B A}-\mathbf{O C}_{\mathbf{1 4}}$, the dilution effect arising from the mixing $\boldsymbol{n}-\mathbf{C}_{\mathbf{5 0}}$ solution already present on the HOPG surface was taken into account (approxi-

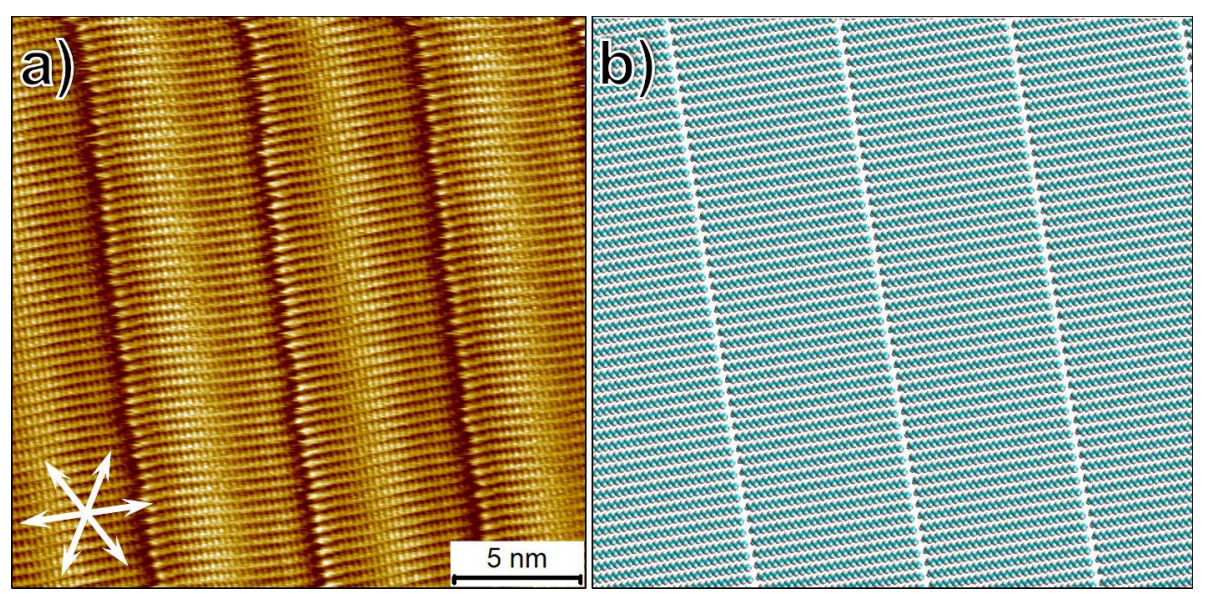

Figure 3: (a) High-resolution STM image of the $\boldsymbol{n}-\mathbf{C}_{\mathbf{5 0}}$ monolayer formed at the 1-phenyloctane-HOPG interface. The white double-headed arrows at the lower left corner of the image represent the three symmetry axes of the graphite lattice. (b) Molecular model for the $\boldsymbol{n}$ - $\boldsymbol{C}_{50}$ monolayer. Imaging conditions: $I_{\text {set }}=200 \mathrm{pA}, V_{\text {bias }}=-0.8 \mathrm{~V}$. 
mately $50 \%$ dilution, since equal volumes of the two solutions were used). Similar precaution was exercised when the premixed solutions were prepared. For BA-OC $\mathbf{1 4}$ the chosen concentration was the one where its self-assembly behavior on bare HOPG is fully known. Thus, a BA-OC $\mathbf{1 4}$ concentration of $3.8 \times 10^{-4} \mathrm{M}$ was selected because the net concentration after mixing is $\approx 1.9 \times 10^{-4} \mathrm{M}$ where polymorph $\mathrm{A}$ was observed at the bare HOPG-1-phenyloctane interface. For $\boldsymbol{n}-\mathbf{C}_{\mathbf{5 0}}$, the chosen optimal concentration was $0.01 \mathrm{mM}$, because at this concentration we observed reasonably high-average domain size and relatively low dynamics within the $\boldsymbol{n}-\mathbf{C}_{\mathbf{5 0}}$ monolayer.

Figure $4 \mathrm{a}-\mathrm{c}$ shows a representative large scale STM image of the HOPG surface upon addition of a $3.8 \times 10^{-4} \mathbf{M ~ B A - O C}_{\mathbf{1 4}}$ solution onto the HOPG surface covered with the $\boldsymbol{n}-\mathbf{C}_{\mathbf{5 0}}$ monolayer. Surprisingly, the large-scale images reveal networks of $\mathbf{B A}-\mathbf{O C}_{\mathbf{1 4}}$ adsorbed atop the $\boldsymbol{n}-\mathbf{C}_{\mathbf{5 0}}$ buffer layer that are reminiscent of the ones observed on bare HOPG in the absence of the buffer layer. At this concentration of $\mathbf{B A}-\mathbf{O C}_{\mathbf{1 4}}$ $\left(\approx 1.9 \times 10^{-4} \mathrm{M}\right)$, we could observe all the three polymorphs atop the $\boldsymbol{n}-\mathbf{C}_{\mathbf{5 0}}$ buffer layer whereas only polymorph A was formed in the absence of the buffer layer. High-resolution STM images were recorded for scrutinizing the self-assembled networks further and to determine the unit cell parameters of the three polymorphs. Figure $4 \mathrm{~d}-\mathrm{f}$ shows high-resolution STM images which reveal that the three structures formed atop the $\boldsymbol{n}-\mathbf{C}_{\mathbf{5 0}}$ buffer layer are exactly the same as those observed when formed directly on top of the HOPG substrate. The unit cell parameters obtained for the structures formed on the buffer layer are comparable to those formed at the 1-phenyloctane-HOPG interface (Table 1).

A notable feature of the high-resolution STM images presented in Figure $4 \mathrm{~d}-\mathrm{f}$ is that the alkyl chains of the BA-OC $\mathbf{1 4}$ molecules within the monolayers formed on top of the buffer layer still appear to be in registry with the graphite symmetry axes. This begs a question whether the $\mathbf{B A - O C} \mathbf{1 4}$ is indeed formed on the top of the buffer layer? To confirm the bilayer formation
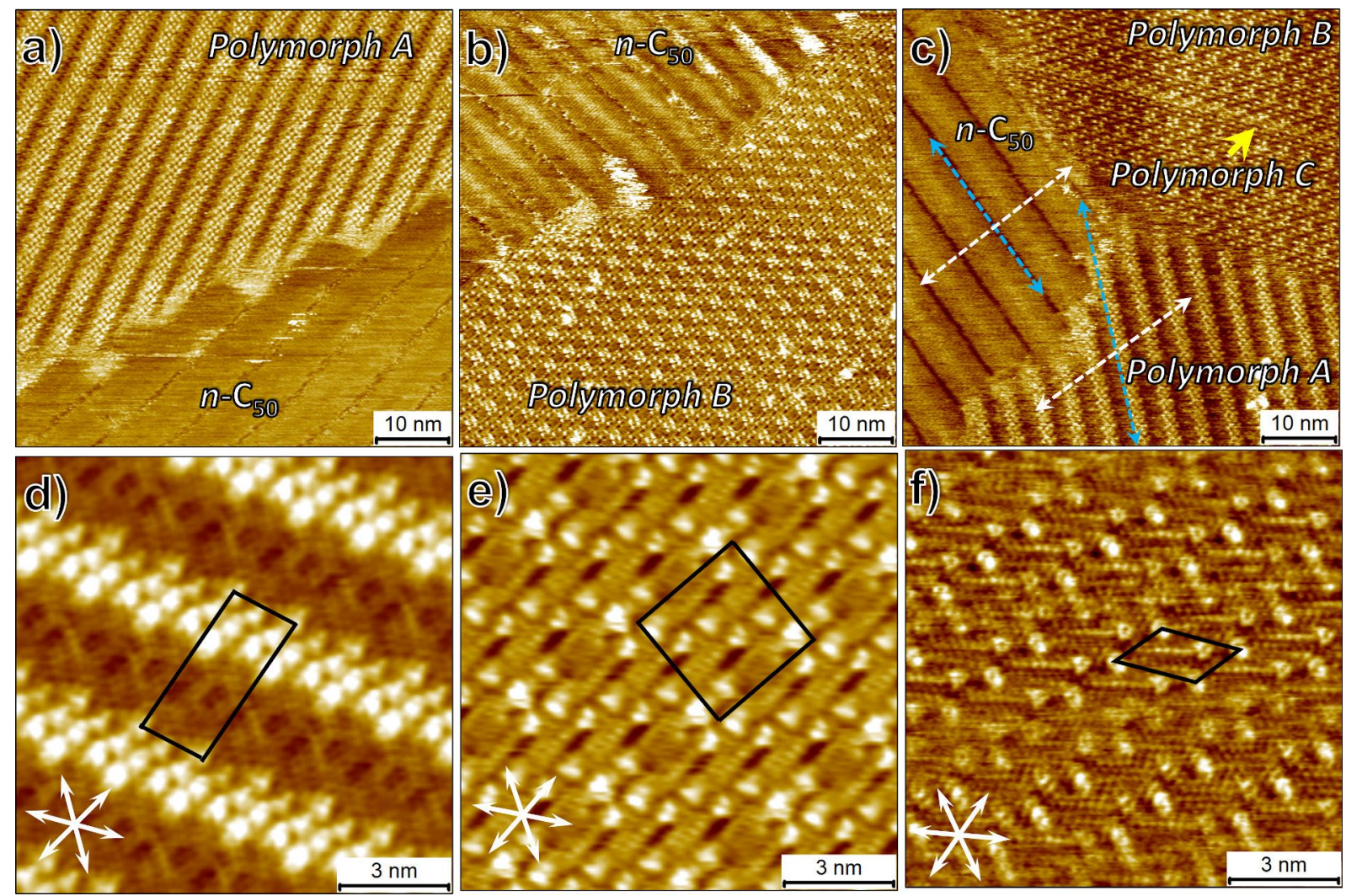

Figure 4: Self-assembled monolayers of $\mathbf{B A}-\mathbf{O C}_{14}$ formed on top of the $\boldsymbol{n}-\mathbf{C}_{50}$ buffer layer at the 1-phenyloctane-HOPG interface. The experiment was carried out using sequential deposition of the two solutions $\left[\mathrm{BA}^{-} \mathrm{OC}_{14}\right]=3.8 \times 10^{-4} \mathrm{M},\left[n-\mathrm{C}_{50}\right]=1.0 \times 10^{-5} \mathrm{M}$. (a-c) Representative large scale STM images showing the formation of polymorph $A$ and polymorph $B$ atop the $n-C_{50}$ buffer layer. (d-f) Representative high-resolution images showing the structure of the polymorph $A$ and polymorph $B$ atop the $\boldsymbol{n}-\mathbf{C}_{50}$ buffer layer. The white double-headed arrows at the lower left corner of the image represent the three symmetry axes of the graphite lattice. Imaging conditions: (a) $I_{\text {set }}=120 \mathrm{pA}, V_{\text {bias }}=-1.2 \mathrm{~V}$; (b) $I_{\text {set }}=110 \mathrm{pA}, V_{\text {bias }}=-1.45 \mathrm{~V}$; (c) $I_{\text {set }}=140 \mathrm{pA}, V_{\text {bias }}=-0.45 \mathrm{~V}$; (d) $I_{\text {set }}=20 \mathrm{pA}, V_{\text {bias }}=-0.17 \mathrm{~V}$; (e) $I_{\text {set }}=110 \mathrm{pA}, V_{\text {bias }}=-1.45 \mathrm{~V}$; (f) $I_{\text {set }}=190 \mathrm{pA}, V_{\text {bias }}=-0.4 \mathrm{~V}$. For additional large-scale images, see Figure S4 in Supporting Information File 1. 
and to rule out phase separation of the two components in 2D, we reversed the sequence of solution deposition. Thus, the solution of $\mathbf{B A}-\mathbf{O C}_{\mathbf{1 4}}$ was added to the bare HOPG substrate first and the monolayer formed (polymorph A) was imaged for a few hours to ascertain full surface coverage. In the second step, $\boldsymbol{n}-\mathbf{C}_{\mathbf{5 0}}$ solution was added to the surface and the STM imaging was resumed. This experiment revealed that $\boldsymbol{n}-\mathbf{C}_{\mathbf{5 0}}$ completely removed the $\mathbf{B A}-\mathbf{O C}_{\mathbf{1 4}}$ monolayer thus confirming that BA-OC $\mathbf{1 4}$ does not compete with $\boldsymbol{n}-\mathbf{C}_{\mathbf{5 0}}$ for adsorption on the HOPG surface thereby also justifying its choice as the buffer layer. Furthermore, certain STM images clearly showed the $\boldsymbol{n}-\mathbf{C}_{\mathbf{5 0}}$ lamellae running underneath the $\mathbf{B A}-\mathbf{O C}_{\mathbf{1 4}}$ monolayer as displayed in Figure 5a-c. The superposition of the STM contrast of the two columns is visible in the STM image which would not be possible if the two monolayers were adsorbed side-by-side on the HOPG surface (see also Figure S5 in Supporting Information File 1). Last but not the least, the controlled nucleation experiments described below, also confirm the formation of the $\mathbf{B A}-\mathbf{O C}_{\mathbf{1 4}}$ islands on top of the buffer layer. The registry of the alkyl chains of $\mathbf{B A}-\mathbf{O} \mathbf{O C}_{\mathbf{1 4}}$ with the graphite lattice can be understood by considering the orientation of the $\mathbf{B A}-\mathbf{O C}_{\mathbf{1 4}}$ islands with respect to the $\boldsymbol{n}-\mathbf{C}_{\mathbf{5 0}}$ lamellae. As depicted in the large scale STM image provided in Figure $4 \mathrm{c}$, the lamellae of polymorph $\mathrm{A}$ are often oriented at approximately $30^{\circ}$ with respect to the $\boldsymbol{n}-\mathbf{C}_{50}$ lamellae (blue dashed arrows). Given that the alkyl chains of $\mathbf{B A}-\mathbf{O} \mathbf{C C}_{\mathbf{1 4}}$ are oriented at an angle of $60^{\circ}$ with respect to its lamella axis, it can be easily deduced that they are aligned along the $\boldsymbol{n}-\mathbf{C}_{\mathbf{5 0}}$ molecular axis as highlighted by the white dashed arrows which indicate the long molecular axes of the $\boldsymbol{n}-\mathbf{C}_{\mathbf{5 0}}$ and the $\mathbf{B A}-\mathbf{O C}_{\mathbf{1 4}}$ molecules (also see Figure S6 in Supporting Information File 1). However, we also discovered that in a few cases the two molecules are not always aligned as is the case in Figure 5a-c.

For $\boldsymbol{n}-\mathbf{C}_{\mathbf{5 0}}$, the chosen optimal concentration was $1.0 \times 10^{-4} \mathrm{M}$, because at this concentration we observed reasonably high average domain size and relatively low dynamics within the $\boldsymbol{n}-\mathbf{C}_{\mathbf{5 0}}$ monolayer. Lowering the concentration of $\mathbf{B A}-\mathbf{O C}_{\mathbf{1 4}}$ further (range $\approx 1.9 \times 10^{-4} \mathrm{M}$ to $9 \times 10^{-5} \mathrm{M}$ ) while keeping the same concentration of $\boldsymbol{n}-\mathbf{C}_{\mathbf{5 0}}$ led to exclusive formation of polymorph A. Large domains of the polymorph A were observed with very little real-time spontaneous nucleation of domains.

Considering the original goal, our pursuit for new 2D polymorphs of $\mathbf{B A}-\mathbf{O C}_{\mathbf{1 4}}$ atop the $\boldsymbol{n}-\mathbf{C}_{\mathbf{5 0}}$ buffer layer did not yield positive results. This also casts a doubt on the extent of the influence of the substrate lattice on the assembly of alkoxybenzoic acids as originally proposed by us [45]. While the observation of similar polymorphs on top of the buffer layer does not necessarily rule out the role of substrate lattice on the assembly, it is certainly not the sole explanation for the peculiar network structure of polymorph A. At this juncture, we believe that the
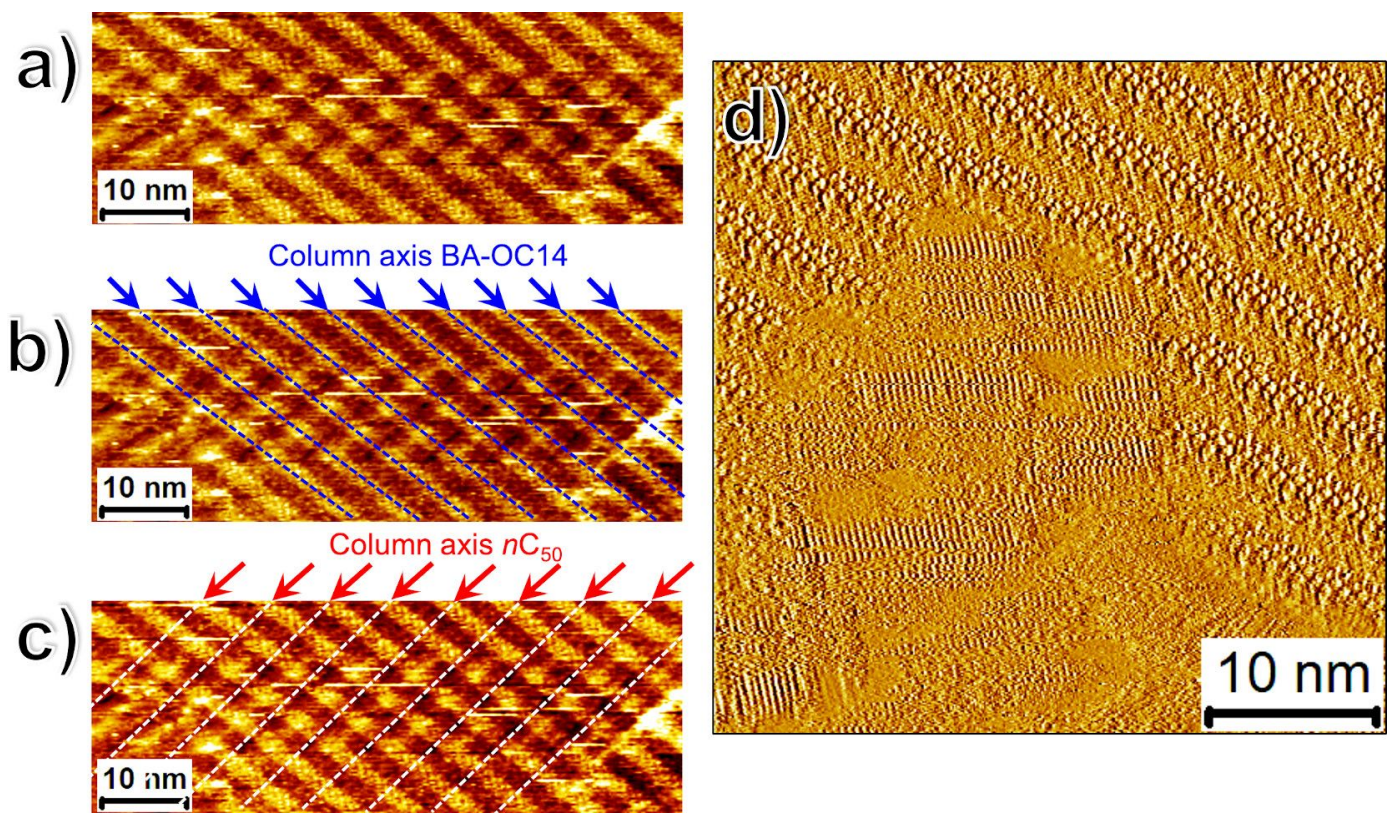

Figure 5: (a) STM image showing the superposition of the $\boldsymbol{n}-\mathbf{C}_{\mathbf{5 0}}$ lamellae with those of BA-OC $\mathbf{1}_{\mathbf{1 4}}$ confirming the bilayer structure of the system. Panels (b) and (c) show the same STM image with colored markers to aid the eye to identify the superimposed STM contrast arising from the columns of benzoic acid (blue dotted lines) and those of pentacontane (white dotted lines) running underneath. For the corresponding large-scale image see Figure S5 in Supporting Information File 1. Imaging conditions: $I_{\text {set }}=130 \mathrm{pA}, V_{\text {bias }}=-0.65 \mathrm{~V}$. (b) STM current image showing the alignment of the chains of the tetradecyloxy chains of $\mathbf{B A}-\mathbf{O C}_{\mathbf{1 4}}$ with the long axes of $\boldsymbol{n}-\mathbf{C}_{\mathbf{5 0}}$ molecules. Imaging conditions: $I_{\text {set }}=90 \mathrm{pA}, V_{\mathrm{bias}}=-0.17 \mathrm{~V}$. 
formation of the characteristic kinked lamellae in the case of 4-alkoxybenzoic acids is a result of a tendency to achieve closepacked assemblies together with the contribution of various other factors, the influence of the substrate lattice being possibly one of them. We have recently reported on similar behavior in the case of brominated alkoxybenzenes where a brickwall type structure was ascribed to a tendency to form close packed networks since the formation of kinks/fractures within the monolayer allowed higher packing densities in the monolayers [47].

While studying the self-assembly of $\mathbf{B A}-\mathbf{O C}_{\mathbf{1 4}}$ on the buffer layers, we discovered a number of interesting aspects of this peculiar interface. As anticipated, the mobility of $\mathbf{B A}-\mathbf{O C}_{\mathbf{1 4}}$ on the buffer layers was significantly higher than that at the 1-phenyloctane-HOPG interface. It was possible to observe the nucleation, the growth as well as the desorption of molecular domains (see Figure S7 in the Supporting Information File 1) which was otherwise not possible for monolayers adsorbed directly on the graphite surface. In the following section we describe our attempts to observe such dynamic phenomena and to induce nucleation of $\mathbf{B A - O C} \mathbf{1 4}$ domains using the STM tip.

\section{STM tip-induced nucleation on-command atop $\boldsymbol{n}$ - $\mathbf{C}_{\mathbf{5 0}}$ buffer layer}

During the course of this investigation, we uncovered that it is possible to enforce the nucleation of small $\mathbf{B A}-\mathbf{O} \mathbf{O C}_{\mathbf{1 4}}$ islands on the buffer layers using the STM tip. Such "on-command" nucleation was achieved using two different stimuli, namely by applying short voltage pulses to the STM tip and by scanning the surface of the buffer layer at higher than normal tunneling currents (Figure 6a). Application of voltage pulses to the STM tip while imaging the $\boldsymbol{n}-\mathbf{C}_{\mathbf{5 0}}$ buffer layer (containing $\mathbf{B A}-\mathbf{O C} \mathbf{1 4}$ in the supernatant) lead to nucleation of small islands of polymorph A of BA-OC $\mathbf{1 4}$. The domain size was typically around $20 \times 20 \mathrm{~nm}$. These domains were found to either grow or shrink upon subsequent STM imaging of the same area. Figure $6 \mathrm{~b}-\mathrm{e}$ shows one such pulse-induced on-command nucleation event. A voltage pulse with a magnitude of $-4.2 \mathrm{~V}$ lasting $1 \mathrm{~ms}$ was applied onto an 'empty' region of the $\boldsymbol{n}-\mathbf{C}_{\mathbf{5 0}}$ buffer layer (marked by an arrow in Figure 6c) which did not have any adsorbed domain of BA-OC $\mathbf{1 4}$. A BA-OC $\mathbf{1 4}$ domain can be seen to appear right after the voltage pulse was applied (white arrow, Figure 6c) and was found to grow in the subsequent scans (white arrows, Figure 6d,e). Such pulse-induced nucleation was possible to carry out by selecting any area of the buffer layer which did not have a $\mathbf{B A}-\mathbf{O C}_{\mathbf{1 4}}$ domain. We also systematically examined the influence of the magnitude and the duration of the voltage pulse on the nucleation events. This exercise revealed that a pulse width of $1 \mathrm{~ms}$ and a minimum re- quired pulse height of $-3.3 \mathrm{~V}$ was necessary for inducing the nucleation of BA-OC $\mathbf{~}_{\mathbf{1 4}}$ domains. The outcome of the pulse-induced nucleation experiment remained the same irrespective of the sign of the pulse.

Scanning small areas of the $\boldsymbol{n}-\mathbf{C}_{\mathbf{5 0}}$ buffer layer (with $\mathbf{B A}-\mathbf{O C}_{\mathbf{1 4}}$ supernatant) at higher tunneling currents and lower bias (typically $-0.05 \mathrm{~V}$ and $0.5 \mathrm{nA}$ ) provided better control over the on-command nucleation process. These parameters are used for obtaining the resolution at which the graphite lattice is visible. Figure 6f shows an STM image of the buffer layer. The area highlighted by the square $(30 \mathrm{~nm} \times 30 \mathrm{~nm})$ was scanned using the aforementioned parameters. In the subsequent scan, the parameters were changed to normal imaging parameters and the size of the scan was restored to the original (zoomed out). The subsequent scan presented in Figure $6 \mathrm{~g}$ reveals the presence of a small domain of $\mathbf{B A}-\mathbf{O C}_{\mathbf{1 4}}$ in the area scanned at the different tunneling parameters. It can also be noticed that the lamellae of the new domain follow the same registry conditions $\left(\approx 30^{\circ}\right.$ with respect to the column axis of $\left.\boldsymbol{n}-\mathbf{C}_{\mathbf{5 0}}\right)$ as described earlier. This means that the tetradecyloxy chains of $\mathbf{B A}-\mathbf{O C}_{\mathbf{1 4}}$ are aligned with the alkane molecules underneath. We note that the 'nucleation on command' experiments always furnished polymorph A.

Quite naturally, one could suspect that the observed nucleation occurs on top of the graphite surface, because under the scanning parameters used, the STM tip is pushed very close to the substrate allowing possible removal of the buffer layer. If this was the case, one would observe gradual removal of the nucleated domain of $\mathbf{B A}-\mathbf{O C}_{\mathbf{1 4}}$ by $\boldsymbol{n}-\mathbf{C}_{\mathbf{5 0}}$ molecules. This was not observed. In fact, in some cases, after inducing the nucleation in this fashion, the newly formed $\mathbf{B A - O C} \mathbf{1 4}$ domain was found to grow rapidly (Figure S8 in Supporting Information File 1). If this would happen in a hypothetical scenario where the $\mathbf{B A}_{\mathbf{O}} \mathbf{O C}_{\mathbf{1 4}}$ domain nucleated directly on HOPG, it would require the displacement of $\boldsymbol{n}-\mathbf{C}_{\mathbf{5 0}}$ molecules by $\mathbf{B A}-\mathbf{O C}_{\mathbf{1 4}}$ molecules which was never observed during the independent control experiments. Similar to the pulse-induced process, the scanninginduced nucleation could also be stimulated a number of times although in some instances (minority cases) no nucleation of BA-OC $_{\mathbf{1 4}}$ domain was observed.

So, what is the mechanism behind the on-command nucleation of BA-OC $\mathbf{1 4}$ on the buffer layers? We propose that the stimuli provided, namely scanning at higher currents and the voltage pulsing, removes the residual layer of $\boldsymbol{n}-\mathbf{C}_{\mathbf{5 0}}$ molecules interacting with the monolayer of $\boldsymbol{n}-\mathbf{C}_{\mathbf{5 0}}$ adsorbed on the surface. Given that the concentration of $\boldsymbol{n}-\mathbf{C}_{\mathbf{5 0}}$ in solution is well above than that required to form a monolayer, it is not unreasonable to consider that there exist excess molecules of $\boldsymbol{n}-\mathbf{C}_{\mathbf{5 0}}$ that interact 


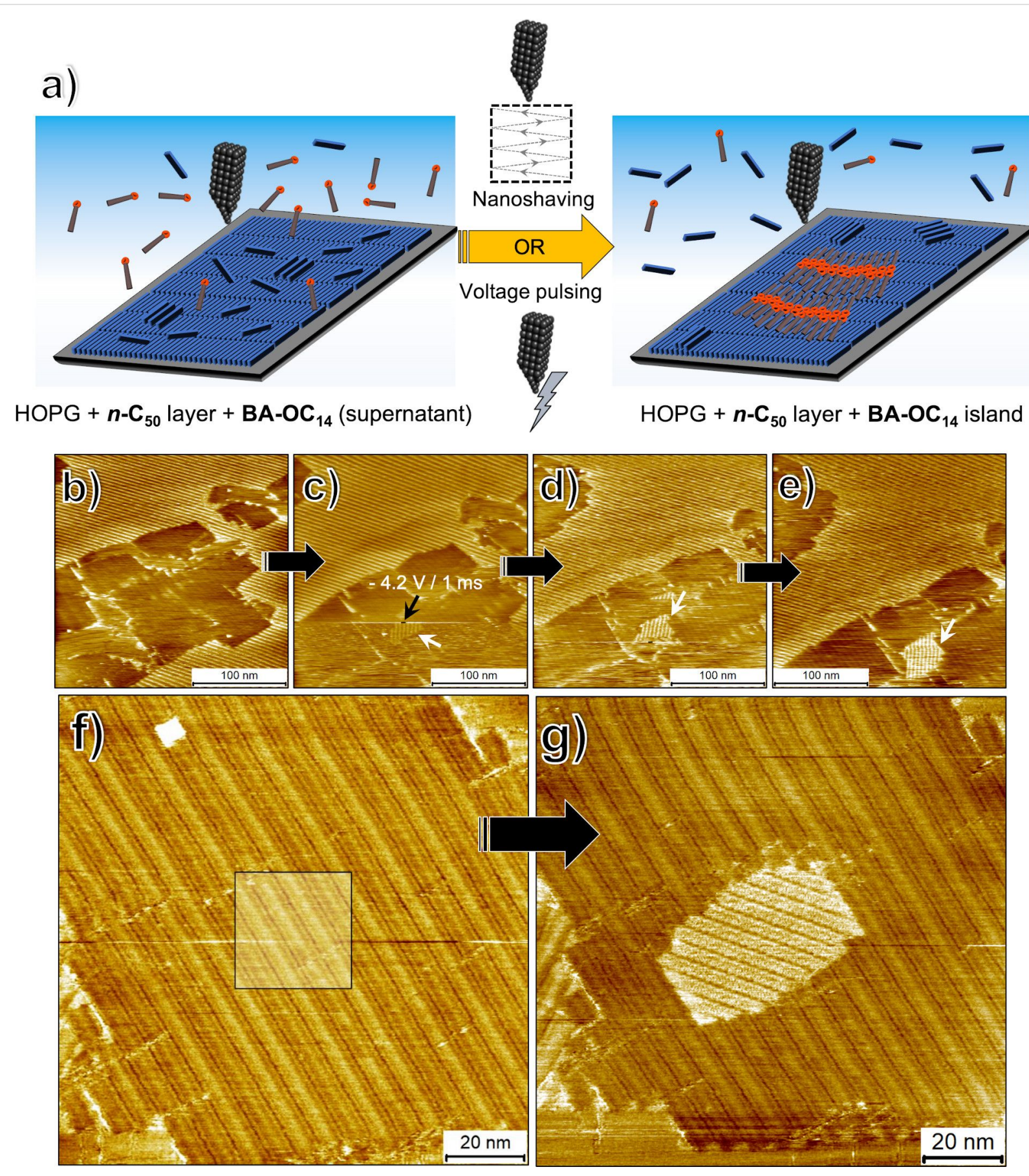

Figure 6: On-command nucleation of BA-OC $\mathbf{C}_{14}$ islands on top of the $\boldsymbol{n}-\mathbf{C}_{50}$ buffer layer. (a) Schematic for the STM-induced nucleation process. (b-e) Sequential STM images where the nucleation of BA-OC $\mathbf{C}_{14}$ islands was achieved using application of voltage pulses to the STM tip. The black arrow in (c) shows the point at which a $-4.2 \mathrm{~V}(1 \mathrm{~ms})$ pulse was applied to the STM tip. The white arrow shows the island of BA-OC ${ }_{14}$ that nucleated immediately after the pulse. As evident in panels $(d, e)$ this island grew in subsequent scans. $(f, g)$ Sequential STM images showing nucleation on-command by bringing the STM tip closer to the surface and scanning at high tunneling current. The square highlighted in (f) was scanned at $-0.05 \mathrm{~V}$ and $0.5 \mathrm{nA}$. After this, the original image size was restored by zooming out. As evident from $(\mathrm{g})$ a new island of $\mathbf{B A}^{-}-\mathbf{O C}_{14}$ is nucleated approximately in the area which was manipulated in the previous scan. Imaging conditions: $(\mathrm{b}-\mathrm{e}) I_{\mathrm{set}}=130 \mathrm{pA}, V_{\text {bias }}=-1.3 \mathrm{~V} ;(\mathrm{f}, \mathrm{g}) I_{\text {set }}=140 \mathrm{pA}, V_{\text {bias }}=$ $-0.7 \mathrm{~V}$.

with the preformed buffer layer. 'Blasting' the surface of the buffer layer with a voltage pulse or 'sweeping' it by bringing the tip closer to the surface removes these excess $\boldsymbol{n}-\mathbf{C}_{\mathbf{5 0}}$ mole- cules thereby allowing the nucleation of $\mathbf{B A - O C} \mathbf{1 4}$ islands which then subsequently grow. The observed relative concentration dependency of nucleation and growth also suggests that 
a higher $\boldsymbol{n}-\mathbf{C}_{\mathbf{5 0}}$ concentration 'blocks' the nucleation of BA-OC $_{14}$ on top of the buffer layer.

\section{Conclusion}

New structures, whether in bulk or on surfaces, are found as long as one keeps looking for them by continuously adjusting experimental conditions that affect (2D) crystallization. Keeping this generally accepted perception in mind, we had set out to alter an important factor that is known to influence selfassembly on solid surfaces, namely the influence of the substrate lattice. We used a monolayer of $\boldsymbol{n}-\mathbf{C}_{\mathbf{5 0}}$ as a 'new' substrate for the self-assembly of $\mathbf{B A}-\mathbf{O C}_{\mathbf{1 4}}$ at the solution-solid interface and anticipated that, given the different lattice constant of the $\boldsymbol{n}-\mathbf{C}_{\mathbf{5 0}}$ layer compared to graphite, we would create possibilities for the formation of new polymorph(s). The selfassembly experiments using the buffer layer however revealed that $\mathbf{B A}-\mathbf{O C}_{\mathbf{1 4}}$ forms the same structural polymorphs in the presence and in the absence of the buffer layer. This indicates that the peculiar network formation, at least in the case of polymorph A, cannot be solely explained by the influence of the substrate lattice. We hypothesize that in the case of alkoxybenzoic acids, a tendency to form close-packed networks supersedes other factors.

The buffer layer however provided a unique platform for selfassembly experiments where the nucleation, growth and ripening of the self-assembled monolayer could be monitored in a more controlled fashion compared to that on the surface of HOPG. The on-command nucleation demonstrated here is an exciting approach to study nucleation of typical systems with precise control on the size and shape of the domains that can be formed using the STM tip. This approach holds a promise as a new platform for investigating elementary stages of selfassembly processes. Despite the lack of discovery of new polymorphs in the present case, the buffer layer strategy could prove fruitful towards identification of new structures in future.

\section{Experimental}

$\boldsymbol{n}-\mathbf{C}_{\mathbf{5 0}}$ was obtained from Sigma-Aldrich (purity $\geq 97 \%$ ) and used without further purification. BA-OC $\mathbf{1 4}$ was synthesized using a protocol previously reported by us [45]. Stock solutions of $\boldsymbol{n}-\mathbf{C}_{\mathbf{5 0}}\left(1.0 \times 10^{-3} \mathrm{M}\right)$ and $\mathbf{B A}-\mathbf{O C}_{\mathbf{1 4}}\left(7.7 \times 10^{-4} \mathrm{M}\right)$ were prepared by dissolving appropriate amounts of solid materials in 1-phenyloctane (Sigma-Aldrich, >99\%). The stock solutions were diluted further with 1-phenyoctane to make concentration series. All STM experiments were performed at room temperature $\left(21-23{ }^{\circ} \mathrm{C}\right)$ using a PicoLE or a PicoSPM (Agilent) machine operating in constant-current mode with the tip immersed in the supernatant liquid. STM tips were prepared by mechanically cutting a Pt/Ir wire $(80 \% / 20 \%$, diameter $0.2 \mathrm{~mm})$. Prior to imaging, a drop of solution was placed onto a freshly cleaved surface of highly oriented pyrolytic graphite (HOPG, grade ZYB, Advanced Ceramics Inc., Cleveland, USA). For experiments involving the $\boldsymbol{n}-\mathbf{C}_{\mathbf{5 0}}$ buffer layer, both sequential deposition and premixing protocols were used. For sequential deposition, a solution of $\boldsymbol{n}-\mathbf{C}_{\mathbf{5 0}}$ was first applied to a freshly cleaved graphite surface. The STM imaging was carried out to ensure full surface coverage of the buffer layer. After this, a drop of BA-OC $\mathbf{1 4}$ solution was applied and the imaging was resumed. The STM experiments were repeated in 2-3 sessions using different tips to check for reproducibility and to avoid experimental artefacts, if any. For voltage pulsing experiments described here, the pulses were applied during the line scan. Each voltage pulse was applied to the substrate after retracting the STM tip at a certain distance (around $1 \mathrm{~nm}$ ) from the surface. The feedback loop was turned off to maintain the separation between the tip and the sample during the period of voltage pulse in order to avoid the tip crash onto the surface. The software used for STM imaging does not log the tunneling current reached during each pulse. For analysis purposes, recording of a monolayer image was followed by imaging the graphite substrate underneath it under the same experimental conditions, except for increasing the current and lowering the bias. The images were corrected for drift via Scanning Probe Image Processor (SPIP) software (Image Metrology ApS), using the recorded graphite images for calibration purposes, allowing a more accurate unit cell determination. The unit cell parameters were determined by examining at least 4 images and only the average values are reported. The images are Gaussian filtered and/or correlation averaged. The imaging parameters are indicated in the figure caption: tunneling current $\left(I_{\text {set }}\right)$, and sample bias $\left(V_{\text {bias }}\right)$. The molecular models were built using HyperchemTM 8.0.1 program.

\section{Supporting Information}

\section{Supporting Information File 1 \\ Additional STM data. \\ [https://www.beilstein-journals.org/bjnano/content/ supplementary/2190-4286-11-113-S1.pdf]}

\section{Acknowledgements}

We thank Prof. Koen Binnemans (Department of Chemistry, KU Leuven) for kindly providing the sample of 4-alkoxybenzoic acid used in this study and Dr. Lander Verstraete for providing the high-resolution STM image of the $\boldsymbol{n}-\mathbf{C}_{\mathbf{5 0}}$ monolayer.

\section{Funding}

This work is financially supported by the Fund of Scientific Research - Flanders (FWO), in part by FWO under EOS 
30489208, and KU Leuven Research Fund (C14/15/053 and C14/19/079).

\section{ORCID ${ }^{\circledR}$ iDs}

Kunal S. Mali - https://orcid.org/0000-0002-9938-6446

Steven De Feyter - https://orcid.org/0000-0002-0909-9292

\section{References}

1. Desiraju, G. R. J. Am. Chem. Soc. 2013, 135, 9952-9967. doi:10.1021/ja403264c

2. Goronzy, D. P.; Ebrahimi, M.; Rosei, F.; Arramel; Fang, Y.; De Feyter, S.; Tait, S. L.; Wang, C.; Beton, P. H.; Wee, A. T. S.; Weiss, P. S.; Perepichka, D. F. ACS Nano 2018, 12, 7445-7481. doi:10.1021/acsnano.8b03513

3. Lackinger, M.; Heckl, W. M. Langmuir 2009, 25, 11307-11321. doi:10.1021/la900785f

4. Tahara, K.; Lei, S.; Adisoejoso, J.; De Feyter, S.; Tobe, Y. Chem. Commun. 2010, 46, 8507-8525. doi:10.1039/c0cc02780d

5. Mali, K. S.; Pearce, N.; De Feyter, S.; Champness, N. R. Chem. Soc. Rev. 2017, 46, 2520-2542. doi:10.1039/c7cs00113d

6. Gutzler, R.; Sirtl, T.; Dienstmaier, J. F.; Mahata, K.; Heckl, W. M.; Schmittel, M.; Lackinger, M. J. Am. Chem. Soc. 2010, 132, 5084-5090. doi:10.1021/ja908919r

7. Blunt, M. O.; Adisoejoso, J.; Tahara, K.; Katayama, K.; Van der Auweraer, M.; Tobe, Y.; De Feyter, S. J. Am. Chem. Soc. 2013, 135, 12068-12075. doi:10.1021/ja405585s

8. Marie, C.; Silly, F.; Tortech, L.; Müllen, K.; Fichou, D. ACS Nano 2010, 4, 1288-1292. doi:10.1021/nn901717k

9. Bellec, A.; Arrigoni, C.; Schull, G.; Douillard, L.; Fiorini-Debuisschert, C.; Mathevet, F.; Kreher, D.; Attias, A.-J.; Charra, F. J. Chem. Phys. 2011, 134, 124702. doi:10.1063/1.3569132

10. Kampschulte, L.; Lackinger, M.; Maier, A.-K.; Kishore, R. S. K.; Griessl, S.; Schmittel, M.; Heckl, W. M. J. Phys. Chem. B 2006, 110, 10829-10836. doi:10.1021/jp057553m

11. Takami, T.; Mazur, U.; Hipps, K. W. J. Phys. Chem. C 2009, 113, 17479-17483. doi:10.1021/jp905223b

12. Zhang, X.; Chen, T.; Chen, Q.; Deng, G.-J.; Fan, Q.-H.; Wan, L.-J. Chem. - Eur. J. 2009, 15, 9669-9673. doi:10.1002/chem.200901618

13. Lackinger, M.; Griessl, S.; Heckl, W. M.; Hietschold, M.; Flynn, G. W. Langmuir 2005, 21, 4984-4988. doi:10.1021/la0467640

14. Ochs, O.; Hocke, M.; Spitzer, S.; Heckl, W. M.; Martsinovich, N.; Lackinger, M. Chem. Mater. 2020, 32, 5057-5065. doi:10.1021/acs.chemmater.0c00827

15. Wang, S.; Zhao, F.; Luo, S.; Geng, Y.; Zeng, Q.; Wang, C. Phys. Chem. Chem. Phys. 2015, 17, 12350-12355. doi:10.1039/c5cp00531k

16. Balandina, T.; Tahara, K.; Sändig, N.; Blunt, M. O.; Adisoejoso, J.; Lei, S.; Zerbetto, F.; Tobe, Y.; De Feyter, S. ACS Nano 2012, 6, 8381-8389. doi:10.1021/nn303144r

17. Katsonis, N.; Marchenko, A.; Fichou, D. J. Am. Chem. Soc. 2003, 125, 13682-13683. doi:10.1021/ja0375737

18. Kudernac, T.; Sändig, N.; Fernandez Landaluce, T.; van Wees, B. J.; Rudolf, P.; Katsonis, N.; Zerbetto, F.; Feringa, B. L. J. Am. Chem. Soc. 2009, 131, 15655-15659. doi:10.1021/ja901718q

19. Ahn, S.; Matzger, A. J. J. Am. Chem. Soc. 2010, 132, 11364-11371. doi:10.1021/ja105039s
20. Lei, S.; Tahara, K.; De Schryver, F. C.; Van der Auweraer, M.; Tobe, Y.; De Feyter, S. Angew. Chem., Int. Ed. 2008, 47, 2964-2968. doi:10.1002/anie.200705322

21. Adisoejoso, J.; Tahara, K.; Lei, S.; Szabelski, P.; Rżysko, W.; Inukai, K.; Blunt, M. O.; Tobe, Y.; De Feyter, S. ACS Nano 2012, 6, 897-903. doi:10.1021/nn204398m

22. Shen, X.; Wei, X.; Tan, P.; Yu, Y.; Yang, B.; Gong, Z.; Zhang, H.; Lin, H.; Li, Y.; Li, Q.; Xie, Y.; Chi, L. Small 2015, 11, 2284-2290. doi:10.1002/smll.201402645

23. Cometto, F. P.; Kern, K.; Lingenfelder, M. ACS Nano 2015, 9, 5544-5550. doi:10.1021/acsnano.5b01658

24. Miao, X.; Xu, L.; Cui, L.; Deng, W. Phys. Chem. Chem. Phys. 2014, 16, 12544-12553. doi:10.1039/c4cp00871e

25. Xu, B.; Yin, S.; Wang, C.; Qu, X.; Zeng, Q.; Bai, C. J. Phys. Chem. B 2000, 104, 10502-10505. doi:10.1021/jp001618y

26. Piot, L.; Marchenko, A.; Wu, J.; Müllen, K.; Fichou, D. J. Am. Chem. Soc. 2005, 127, 16245-16250. doi:10.1021/ja0548844

27. Rohr, C.; Balbás Gambra, M.; Gruber, K.; Höhl, C.; Malarek, M. S.; Scherer, L. J.; Constable, E. C.; Franosch, T.; Hermann, B. A. Chem. Commun. 2011, 47, 1800-1802. doi:10.1039/c0cc03603j

28. Li, Y.; Liu, L.; Subramani, R.; Pan, Y.; Liu, B.; Yang, Y.; Wang, C.; Mamdouh, W.; Besenbacher, F.; Dong, M. Chem. Commun. 2011, 47, 9155-9157. doi:10.1039/c1cc11443c

29. Lei, S. B.; Wang, C.; Yin, S. X.; Wang, H. N.; Xi, F.; Liu, H. W.; Xu, B.; Wan, L. J.; Bai, C. L. J. Phys. Chem. B 2001, 105, 10838-10841. doi:10.1021/jp0105701

30. Lei, S. B.; Wang, C.; Yin, S. X.; Xu, Q. M.; Bai, C. L. Surf. Interface Anal. 2001, 32, 253-255. doi:10.1002/sia.1048

31. Lei, S.; Wang, C.; Wan, L.; Bai, C. J. Phys. Chem. B 2004, 108, 1173-1175. doi:10.1021/jp037457q

32. Yang, X.; Krieger, I.; Lüftner, D.; Weiß, S.; Heepenstrick, T.; Hollerer, M.; Hurdax, P.; Koller, G.; Sokolowski, M.; Puschnig, P.; Ramsey, M. G.; Tautz, F. S.; Soubatch, S. Chem. Commun. 2018, 54, 9039-9042. doi:10.1039/c8cc03334j

33. Forker, R.; Kasemann, D.; Dienel, T.; Wagner, C.; Franke, R.; Müllen, K.; Fritz, T. Adv. Mater. (Weinheim, Ger.) 2008, 20 , 4450-4454. doi:10.1002/adma.200801112

34. Rastgoo-Lahrood, A.; Björk, J.; Lischka, M.; Eichhorn, J.; Kloft, S.; Fritton, M.; Strunskus, T.; Samanta, D.; Schmittel, M.; Heckl, W. M.; Lackinger, M. Angew. Chem., Int. Ed. 2016, 55, 7650-7654. doi:10.1002/anie.201600684

35. Rastgoo-Lahrood, A.; Lischka, M.; Eichhorn, J.; Samanta, D.; Schmittel, M.; Heckl, W. M.; Lackinger, M. Nanoscale 2017, 9, 4995-5001. doi:10.1039/c7nr00705a

36. Sakaguchi, H.; Matsumura, H.; Gong, H.; Abouelwafa, A. M. Science 2005, 310, 1002-1006. doi:10.1126/science.1117990

37. Sakaguchi, H.; Matsumura, H.; Gong, H. Nat. Mater. 2004, 3, 551-557. doi:10.1038/nmat1176

38. Nirmalraj, P.; Thompson, D.; Molina-Ontoria, A.; Sousa, M.; Martín, N.; Gotsmann, B.; Riel, H. Nat. Mater. 2014, 13, 947-953. doi:10.1038/nmat4060

39. Neff, J. L.; Götzen, J.; Li, E.; Marz, M.; Hoffmann-Vogel, R. Beilstein J. Nanotechnol. 2012, 3, 186-191. doi:10.3762/bjnano.3.20

40. Repp, J.; Meyer, G.; Paavilainen, S.; Olsson, F. E.; Persson, M. Science 2006, 312, 1196-1199. doi:10.1126/science.1126073

41. Hirjibehedin, C. F.; Lutz, C. P.; Heinrich, A. J. Science 2006, 312, 1021-1024. doi:10.1126/science.1125398 
42. Rau, I. G.; Baumann, S.; Rusponi, S.; Donati, F.; Stepanow, S.;

Gragnaniello, L.; Dreiser, J.; Piamonteze, C.; Nolting, F.;

Gangopadhyay, S.; Albertini, O. R.; Macfarlane, R. M.; Lutz, C. P.;

Jones, B. A.; Gambardella, P.; Heinrich, A. J.; Brune, H. Science 2014,

344, 988-992. doi:10.1126/science.1252841

43. Groszek, A. J. Proc. R. Soc. London, Ser. A 1970, 314, 473-498. doi:10.1098/rspa.1970.0019

44. Ilan, B.; Florio, G. M.; Hybertsen, M. S.; Berne, B. J.; Flynn, G. W. Nano Lett. 2008, 8, 3160-3165. doi:10.1021/nl8014186

45. Mali, K. S.; Lava, K.; Binnemans, K.; De Feyter, S. Chem. - Eur. J. 2010, 16, 14447-14458. doi:10.1002/chem.201001653

46. McCrone, W. C. Polymorphism. In Physics and Chemistry of the Organic Solid State; Fox, D.; Labels, M. M.; Weissberger, M., Eds.; Wiley Interscience: New York, NY, USA, 1965; Vol. 11, pp 725-767.

47. Mukherjee, A.; Sanz-Matias, A.; Velpula, G.; Waghray, D.; Ivasenko, O.; Bilbao, N.; Harvey, J. N.; Mali, K. S.; De Feyter, S. Chem. Sci. 2019, 10, 3881-3891. doi:10.1039/c8sc04499f

\section{License and Terms}

This is an Open Access article under the terms of the Creative Commons Attribution License (https://creativecommons.org/licenses/by/4.0). Please note that the reuse, redistribution and reproduction in particular requires that the authors and source are credited.

The license is subject to the Beilstein Journal of

Nanotechnology terms and conditions:

(https://www.beilstein-journals.org/bjnano)

The definitive version of this article is the electronic one which can be found at: https://doi.org/10.3762/bjnano.11.113 